\title{
Effects of the Central Density Profile and the Stellar Mass Function of Merging Galaxies on Eccentricities and Coalescence Times of Supermassive Black Hole Binaries.
}

\author{
Fazeel Mahmood Khan ${ }^{1}$, Peter Berczik ${ }^{2,3,4}$ and Andreas Just ${ }^{4}$ \\ ${ }^{1}$ Department of Space Science, Institute of Space Technology, P. O. Box 2750 Islamabad, \\ Pakistan \\ ${ }^{2}$ National Astronomical Observatories of China, Chinese Academy of Sciences, Beijing, China \\ ${ }^{3}$ National Academy of Sciences of Ukraine, Main Astronomical Observatory, Kyiv, Ukraine \\ ${ }^{4}$ Astronomisches Rechen-Institut, Zentrum für Astronomie, Univ. of Heidelberg, \\ Mönchhof-Strasse 12-14, 69120 Heidelberg, Germany
}

\begin{abstract}
If able to coalesce in a Hubble time, Supermassive Black hole (SMBH) binaries are very promising sources of gravitational waves $(\mathrm{GW})$. Our earlier studies have shown that SMBH binaries coalesce in post-merger galactic nuclei having triaxial or axisymmetric geometry in a few billion years. In this study, we model the complete evolution of SMBH binaries formed as a result of galaxy mergers having central density profiles that vary from shallow to very steep including a stellar mass function. Energy and angular momentum loss due to GW emission is taken into account using the post-Newtonian approximation. We carry out ten such simulations for each central density profile. The eccentricity of the SMBH binaries remain very high in shallow cusps and decrease systematically for steeper cusps. The coalescence times range from 0.6 to $1.5 \mathrm{Gyr}$ with shorter times for steeper profiles. Typical coalescence times less than a Gyr strengthen our expectation that SMBH binaries should be very promising sources of GW radiation over a wide redshift range.
\end{abstract}

Keywords. Stellar dynamics, black hole physics, Galaxies: kinematics and dynamics, Galaxy: center.

\section{Introduction}

Galaxy centres host Supermassive Black Holes (SMBHs), with masses from $10^{6}-10^{10} M_{\odot}$ (e.g., Kormendy \& Ho 2013). Supermassive Black Hole binaries form as a result of the merger of two galaxies and evolve in three distinct phases, namely dynamical friction, three-body scattering of stars and gravitational wave (GW) emission (Begelman et al. 1980). Dynamical friction efficiently shrinks the separation of the binary SMBHs depending on the mass of the SMBHs and the background density profile of stars (Just et al. 2011) until the binary becomes "hard" at a separation $a \sim a_{\mathrm{h}}$, where $a_{\mathrm{h}}$ is of the order of a few pc for SMBH binaries. Subsequent evolution is caused by three-body scattering of stars by the SMBH binary on intersecting orbits. It is well known that in spherical galaxy models the 2-body relaxation-driven supply of stars on centrophyllic orbits is very limited leading to a stalling of the binary evolution beyond this point famously known as the "Final Parsec Problem" (Milosavljević \& Merritt 2003). Since about a decade, studies of SMBH binary evolution and their coalescence are of prime interest for people interested in a direct detection of GWs. SMBH binaries in the third phase of evolution produce the loudest bursts of GWs detectable to detectors such as eLISA provided SMBHs shrink to such small separations. 


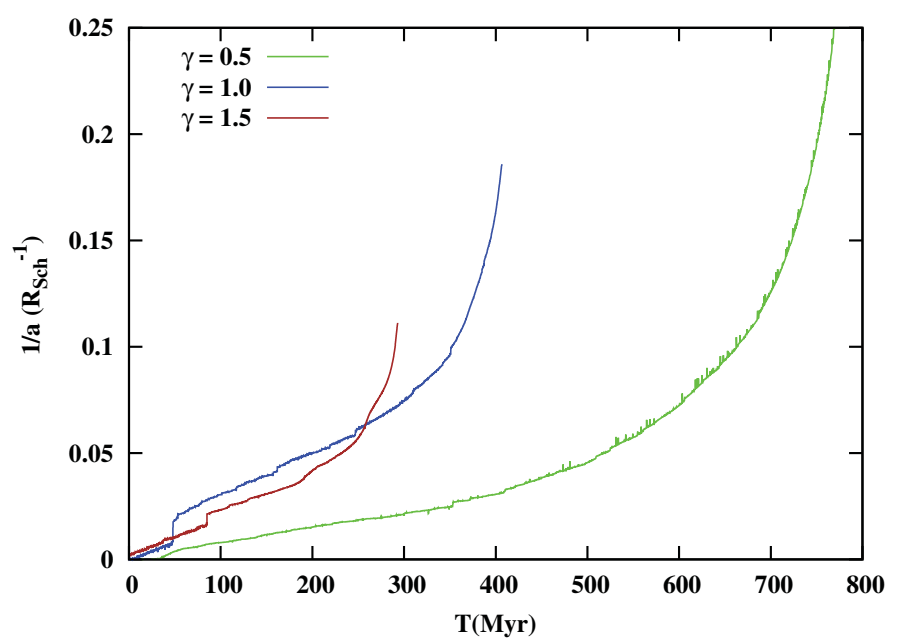

Figure 1. Inverse semi-major axis (in Schwarzschild radii) for selected runs of each $\gamma$.

Vasiliev et al. (2014) witnessed an $N$-dependent evolution of binary's separation in axisymmetric models and concluded that axisymmetry alone does not solve the FPP. However, in more realistic studies of SMBH binary evolution in mergers of spherical bulges of galaxies with equal mass (Khan et al. 2011; Preto et al. 2011), a range of mass ratios (Khan et al. 2012), and of axisymmetric systems (Khan et al. 2013) a significantly faster and $N$-independent SMBH binary evolution was found. Only a very small deviation from axisymmetry close to the sphere of influence of the SMBH binary may suffices to overcome the FPP ( $\mathrm{Li}$ et al. 2014). The estimated SMBH binary coalescence times are as short as 1-2 Gyr for shallow cusp elliptical galaxies and 0.5-1 Gyr for steep cusp spiral galaxies. Hardening rates of binaries in merger simulations seem to match reasonably well with those predicted by scattering theory for full loss cone (Sesana \& Khan 2015). Recently, Vasiliev et al. (2015) also confirmed that triaxial equilibrium models can accommodate SMBH binary coalescence in less than a billion year. Adding rotation to the system accelerates the binary evolution (Holley-Bockelmann \& Khan (2015)).

\section{SMBH Binary Evolution in Mergers of Galaxies Having Different Density Profiles and a Stellar Mass Function}

Here we present the first results of our galaxy merger study, where we follow the complete evolution of SMBHs from the merger of galaxies separated by more than 10 effective radii to the merger of $\mathrm{SMBHs}$ by invoking post-Newtonian $(\mathrm{PN})$ terms up to order 3.5 in the equation of motion of the SMBH binary. Merging galaxies with a 1:4 mass ratio containing central SMBHs with $0.5 \%$ of the galaxy mass according to the M- $\sigma$ relation are realized with Dehnen profiles with three different values of the inner density slope $\gamma=0.5,1.0,1.5$. Each of them we generate nine galaxies with different random seeds for galaxies and stellar mass function. We use the direct $N$-body parallel code $\phi$-GRAPE/GPU (Harfst et al. 2007) on the Laohu cluster of the National Astronomical Observatories of Chinese Academy of Sciences to perform our numerical studies.

Figure 1 shows the inverse semi-major axis evolution for selected three runs for each $\gamma$ of merging galaxies. For shallow cusp galaxies coalescence times are $\sim 1$ Gyr due to high eccentricities in range 0.8-0.95. For steep cusps, eccentricities are low (0.2-0.5), but hardening rates are very high due to the large stellar density resulting in a short 
coalescence time in the range $0.2-0.4 \mathrm{Myr}$. For intermediate case both eccentricities and hardening rates have in between values resulting in a coalescence time $\sim 0.5 \mathrm{Gyr}$. We also conclude that key factors in the coalescence time are the background density of stars and the binary eccentricity, the addition of the stellar mass function only contributes up to $30 \%$. Our studies suggest that for a broad range of density profiles of merging galaxies, coalescence of SMBHs happen within a Gyr after the merger of galaxies leading to a scenario of prompt coalescence of SMBHs in merger remnant.

\section{References}

Begelman, M. C., Blandford, R. D., \& Rees, M. J. 1980, Nature, 287, 307

Harfst, S., Gualandris, A., Merrit, D., et al. 2007, New Astron., 12, 357

Holley-Bockelmann, K. \& Khan, F. M. 2015, ApJ, 810, 139

Just, A., Khan, F. M., Berczik, P., Ernst, A., \& Spurzem, R. 2011, MNRAS, 411, 653

Khan, F. M., Just, A., \& Merritt, D. 2011, ApJ, 732, 89

Khan, F. M., Preto, M., Berczik, P., et al. 2012, ApJ, 749, 147

Khan, F. M., Holley-Bockelmann, K., Berczik, P., \& Just, A. 2013, ApJ, 773, 100

Kormendy, J. \& Ho, L. C. 2013, ARA\&A, 51, 511

Li, B., Holley-Bockelmann, K., \& Khan, F. M. 2015, ApJ, 811, 25

Milosavljević, M. \& Merritt, D. 2003, ApJ, 596, 860

Preto, M., Berentzen, I. Berczik, P., \& Spurzem, R. 2011, ApJ, 732, L26

Sesana, A. \& Khan, F. M. 2015, MNRAS, 454, L66

Vasiliev, E., Antonini, F., \& Merritt, D. 2014, ApJ, 785, 163

Vasiliev, E., Antonini, F., \& Merritt, D. 2015, ApJ, 810, 49 